\title{
Fatores relacionados à prática do tabagismo entre adolescentes e jovens em uma comunidade indígena no estado de Roraima
}

\author{
Factors related to smoking among adolescents and youth in an indigenous community in \\ the state of Roraima
}

Factores relacionados con el tabaquismo en adolescentes y jóvenes de una comunidad
indígena del estado de Roraima

Maria de Fátima da Silva André ${ }^{1}$, Ana Paula Barbosa Alves ${ }^{1 *}$, Hosana Carolina dos Santos Barreto ${ }^{1}$, Simone Lopes de Almeida ${ }^{1}$, Francilene dos Santos Rodrigues ${ }^{1}$, Paula Tainá Barbosa Alves ${ }^{1}$, Fabrício Barreto ${ }^{1}$, Paulo Victor Barbosa Alves ${ }^{1}$, Eliseu Adilson Sandri ${ }^{1}$, Rosielma Barroso da Silva ${ }^{1}$.

\section{RESUMO}

Objetivo: Conhecer os fatores relacionados ao tabagismo entre homens adolescentes e jovens em uma comunidade indígena do estado de Roraima. Métodos: Tratou-se de uma pesquisa exploratória, descritiva de abordagem qualitativa. O local da pesquisa foi na comunidade indígena em um município de Roraima. A coleta de dados utilizada foi a entrevista semiestruturada, o público-alvo foram adolescentes e jovens homens moradores da comunidade. Os dados foram transcritos para tabelas em Excel para facilitar as análises dos resultados. Resultados: Os participantes referiram que experimentaram e continuam consumindo cigarros ou tabaco por influência dos amigos. E o segundo fator mais forte foi o consumo de bebida que favorece, 0 desejo em consumir o cigarro. $E$ o terceiro seria a curiosidade, o quarto seria o desejo e o quinto referem que fumam, porque os seus pais fumam, o que facilita o seu consumo. Conclusão: $O$ tabagismo é elevado entre os jovens e adolescentes, e os principais fatores relacionados correspondem: à influência dos amigos, para ser aceito no grupo, como forma de socialização; o uso de bebidas alcoólicas; a curiosidade, a vontade de conhecer algo novo; o desejo por prazer e relaxamento; sensação de poder por infringir regras; ociosidade; pais fumantes; problemas familiares, entre outros.

Palavras-chave: Tabagismo, Saúde, Adolescente, Povos indígenas.

\begin{abstract}
Objective: To know the factors related to smoking among adolescent and young men in an indigenous community in the state of Roraima. Methods: This was an exploratory, descriptive research with a qualitative approach. The research was carried out in an indigenous community in a municipality of Roraima. The data collection used was a semi-structured interview, and the target audience was adolescents and young men living in the community. The data were transcribed into Excel tables to facilitate the analysis of the results. Results: Participants reported that they have tried and continue to use cigarettes or tobacco because of the influence of friends. And the second strongest factor was the drinking that favors, the desire to consume cigarettes. And the third would be curiosity, the fourth would be desire, and the fifth would be that they smoke because their parents smoke, which facilitates their consumption. Conclusion: Smoking is high among young people and teenagers, and the main factors related to it are: the influence of friends, to be accepted in the group, as a form of socialization; the use of alcoholic beverages; curiosity, the desire to know something new; the desire for pleasure and relaxation; feeling of power by breaking rules; idleness; smoking parents; family problems, among others.
\end{abstract}

Keywords: Smoking, Health, Adolescent, Indigenous peoples.

1 Universidade Federal de Roraima, Boa Vista - RR. *E-mail: paula.alves@ufrr.br

Financiamento PRPPG - UFRR, por meio do processo de seleção estabelecido pelo Edital 06/2021 Pró-Pesquisa - Apoio à Publicação Científica.

SUBMETIDO EM: 1/2022

ACEITO EM: 1/2022

PUBLICADO EM: 2/2022 


\section{RESUMEN}

Objetivo: Conocer los factores relacionados con el tabaquismo entre adolescentes y jóvenes varones de una comunidad indígena del estado de Roraima. Métodos: Se trata de una investigación exploratoria y descriptiva con un enfoque cualitativo. El lugar de la investigación fue en una comunidad indígena en un municipio de Roraima. La recolección de datos utilizada fue la entrevista semiestructurada, el público objetivo fueron los adolescentes y los hombres jóvenes que viven en la comunidad. Los datos se transcribieron en tablas de Excel para facilitar el análisis de los resultados. Resultados: Los participantes informaron de que han probado y siguen consumiendo cigarrillos o tabaco por influencia de sus amigos. Y el segundo factor más fuerte fue el consumo de bebida que favorece, el deseo de consumir cigarrillos. Y la tercera sería la curiosidad, la cuarta el deseo y la quinta referida a que fuman porque sus padres fuman, lo que facilita su consumo. Conclusión: El tabaquismo es alto entre los jóvenes y adolescentes, y los principales factores relacionados corresponden a: la influencia de los amigos, para ser aceptados en el grupo, como forma de socialización; el uso de bebidas alcohólicas; la curiosidad, el deseo de conocer algo nuevo; el deseo de placer y relajación; la sensación de poder al romper las reglas; la ociosidad; los padres fumadores; los problemas familiares, entre otros.

Palabras clave: Fumar, Salud, Adolescente, Pueblos indígenas.

\section{INTRODUÇÃO}

O tabagismo é considerado uma pandemia silenciosa, porque vem causando a morte, anualmente, de milhares de pessoa em todo o mundo, também é uma das principais causas de mortalidade evitáveis (ALVES EC, et al., 2014). A Organização Mundial da Saúde (OMS), (World Health Organization (WHO), em inglês) alerta que 8 milhões de pessoas morrem ao ano em todo o mundo. Entre estes cerca de 7 milhões dessas mortes são em decorrência do uso direto do tabaco, e por volta de 1,2 milhões de mortes são implicações da exposição de não fumantes ao fumo passivo (WHO, 2020).

O Instituto Nacional de Câncer (INCA), afirma que essa enfermidade afeta as pessoas que fumam, e as que convivem com fumantes, sendo as crianças as maiores vítimas, pois são mais vulneráveis para incidência de problemas respiratórios, em comparação com aquelas em que os familiares não fumam (INCA, 2020). A Pesquisa Nacional de Saúde do Escolar (PeNSE) realizada no Brasil, documentou que $30 \%$ dos jovens entre 13 e 15 anos começaram a fumar aos 12 anos de idade (BARRETO SM, et al., 2014).

Borraci RA e Mulassi AH (2015) afirmam que hábitos adquiridos na adolescência, costumam ser mantidos na idade adulta e são mais difíceis de serem modificados. Os adolescentes, na busca pela aceitação social, desejam com a prática do tabagismo sua afirmação como adultos, devido ao seu modo de ser e atitudes tornam-se os mais vulneráveis para a publicidade da indústria tabagista (INCA, 2020).

O Brasil se destaca entre os países que apresentam leis e tratamento ao tabagismo. Assim, esse assunto é muito importante para todos, principalmente para os profissionais da área da saúde (FILHOC BB, et al., 2021). Silva ST, et al. (2014) afirmam que as iniciativas governamentais são importantes para o combate do tabagismo no Brasil, e essas ações podem favorecer a diminuição da exposição de crianças, adolescentes, jovens e adultos ao tabaco. Estratégias como o projeto "Fala Sério" e os programas "Saber Saúde", "Saúde na Escola" e "Unidades de Saúde Livres do Cigarro - Saúde e Coerência" podem fomentar comportamentos mais salutares.

A definição de adolescência e juventude mostra-se como uma tarefa complexa, em razão de que essas etapas estão relacionadas a diferentes ciclos do desenvolvimento humano e as experiências de cada pessoa. Estando envolvidos nesses processos da vida, a maturidade física, cognitiva, emocional, que é determinada por fatores biológicos, genéticos, psicológicos, socioculturais, porém raramente alcançam a proporção real da mudança da infância para a fase adulta, no nosso ciclo da vida humano (KONKIEWITZ EC, 2013).

A OMS define adolescente como os sujeitos que se encontram entre os 10 e os 19 anos de idade. Podese decompô-la em duas etapas: "primeira adolescência", que decorre entre os 10 e os 14 anos e uma "segunda adolescência", que sucede entre os 15 e os 19 anos. Esta fase é caracterizada por um transcurso complexo de crescimento, desenvolvimento biopsicossocial, expressa por meio de mudanças anatômicas, fisiológicas, psicológicas e sociais. E determina juventude (youth, em inglês) como a fase entre os 15 e 24 
anos de idade, esse discernimento tem a finalidade para uso estatístico e político. Ainda utiliza a noção de jovens adultos para as pessoas de 20 aos 24 anos de idade, mas afirma que cada nação pode ter sua própria definição (WHO,1986).

No Brasil, o Estatuto da Criança e do Adolescente estabelece que a adolescência pertence a faixa etária entre 12 aos 18 anos incompletos (BRASIL, 1990). Com a Lei $n^{\circ} 12.852$ de agosto de 2013, que estabelece o regulamento da juventude sobre as garantias dos jovens, os fundamentos e orientações das políticas públicas para juventude e o Sistema Nacional de Juventude (SINAJUVE) são classificados jovens os indivíduos com idade entre 15 e 29 anos de idade (BRASIL, 2013). Compreender essa fase da vida apenas pela perspectiva cronológica de faixa etária é ser reducionista. Esta fase da vida é experimentada de forma plural para cada indivíduo, e para seus grupos sociais suscitando distintos julgamentos e perspectivas do que é ser um indivíduo adolescente (ASSIS SG, et al., 2020).

Hoje em dia, empresas que vendem cigarros têm se dedicado à venda aos adolescentes, uma vez que estes representam os seus novos consumidores. Embora o Estatuto da Criança e do Adolescente (BRASIL, 1990), em seu artigo 81, em que dispõe sobre a proibição da venda para menores de 18 anos, de substâncias com risco de criar dependência física ou psíquica. Ainda assim, em muitos estabelecimentos comerciais são vendidos maços de cigarros às crianças e adolescentes (BUENO SR, 2011).

Neste contexto, a educação como modelo pedagógico para a promoção da saúde se caracteriza como meio de prevenção primária para reduzir a demanda ao uso de drogas. Assim, pretende-se estimular novos pensamentos e, igualmente, favorecer a prevenção ao tabagismo entre os adolescentes e jovens indígenas. Neste sentido, observa-se a relevância desse estudo, pois poderá contribuir com a comunidade na busca de estratégias de prevenção do tabagismo. Portanto, o objetivo geral deste estudo foi conhecer os fatores relacionados ao tabagismo entre jovens e adolescentes indígenas.

\section{MÉTODOS}

Trata-se de uma pesquisa exploratória, descritiva de abordagem qualitativa, que foi submetida ao Comitê de Ética em Pesquisa em Seres Humanos da Universidade Federal de Roraima (CEP/UFRR), e a Comissão Nacional de Ética em Pesquisa (CONEP), seguindo as normas do Conselho Nacional de Saúde do Ministério da Saúde (MS) explicitadas na resolução nº 466 de 12 de dezembro de 2012, que recebeu o parecer de aprovado, $n^{\circ}: 4.356 .412$, com a CAAE de $n^{\circ}$ : 31067820.3.0000.5302.

Este estudo foi motivado a partir da realização do Estágio I, do Curso de Gestão em Saúde Coletiva Indígena em que foi realizado um "Diagnóstico Situacional de Saúde". Neste período, os moradores e as lideranças da comunidade expressaram que o maior problema de saúde era o tabagismo entre adolescentes e jovens. Diante do exposto, a questão que norteou a pesquisa foi: quais são os fatores relacionados à prática do tabagismo entre os adolescentes e jovens indígenas?

O local da pesquisa foi uma comunidade indígena, em um município há $320 \mathrm{~km}$ da capital de Boa VistaRR. O universo populacional do estudo é constituída por 212 adolescentes e jovens moradores da comunidade. A amostra populacional foi de 100 adolescentes e jovens, e teve seu cálculo baseado na fórmula de Gil AC (2018), para populações finitas, que analisa o tamanho necessário dos participantes do estudo. Essa amostra foi baseada para uma população finita de 212 adolescentes e jovens. Porém, foi possível considerar as respostas de um total de 113 pessoas (73 adolescentes e 40 jovens) com faixa etária entre 12 a maiores de 18 anos de idade, todos do sexo masculino. O erro amostral obteve uma margem de $5 \%$ e uma confiança de $95 \%$.

Os critérios de inclusão foram os adolescentes e jovens entre 12 a maiores de 18 anos, que falavam e liam em português e que assinaram o Termo de Consentimento Livre e Esclarecido (TCLE) para os maiores de 18 anos participantes, pais e responsáveis, e o Termo de Assentimento Livre e Esclarecido (TALE) para os jovens entre 12 anos e menores de 18 anos, em que os responsáveis pelo menor, autorizaram a participação no estudo. Foram excluídas do estudo as pessoas que não falavam ou não liam em português e os menores de 12 anos de idade. 
A coleta de dados aconteceu no período de 24/10/2020 a 24/11/2020. Nas atividades foram tomadas todas as condutas necessárias (utilização de máscaras por todos os participantes, mantendo o distanciamento de 2 metros, com disponibilização de álcool em gel para higienização das mãos e superfícies) respeitando os protocolos de segurança devido ao período da pandemia do Coronavírus. As técnicas de coleta de dados utilizadas foram: as entrevistas com o uso de questionários semiestruturados e à observação participante para conseguir expressar o percentual de fumantes e como se iniciou à prática do tabagismo entre os participantes do estudo.

Os encontros e as entrevistas foram feitos na própria comunidade, visto que uma das pesquisadoras é moradora da comunidade e pertence ao povo Macuxi (etnia indígena), e recebeu autorização de toda comunidade e suas lideranças. As respostas dos participantes do estudo (adolescentes, jovens e pais) foram gravadas e as observações foram registradas no diário de campo. Os dados foram transcritos para tabelas em Excel para facilitar as análises dos resultados.

As resposta das entrevistas foram organizadas e analisadas pelo método estatístico de medidas de síntese (porcentagens, índices e médias). Os resultados foram divididos em dois pontos importantes: o percentual de adolescentes e jovens que são fumantes do cigarro comercial na comunidade; e os fatores relacionados à prática do tabagismo entre os adolescentes e jovens.

\section{RESULTADOS}

A comunidade indígena tem a sua própria forma de organização sociopolítica que são essenciais para a proteção e desenvolvimentos de seu povo. As tomadas de decisões são feitas de acordo com as lideranças e a comunidade, como por exemplo, fazer planejamento dos trabalhos comunitários e individuais. A comunidade tem seus modos de vida particulares, utilizam a terra para retirar todos os seus alimentos e repassam seus conhecimentos para as gerações futuras, em que estão incluídas: as línguas maternas, sua medicina tradicional, suas tradições, que são suas maiores riquezas.

A população total da comunidade indígena é de 657 habitantes, sendo dividida em 105 famílias, pertencentes às etnias Macuxi e Wapichana. Possui uma escola estadual e municipal. É administrada por dois Tuxauas. O termo Tuxaua para os indígenas significa a principal liderança em seu território, coopera para ordem, e uniformidade dos modos de vida cotidiana e representação de sua comunidade ou povo frente a outros povos (CARIOCA AT, et al., 2021).

Em relação à prática do tabagismo nosso estudo está relacionado ao consumo do cigarro comum ou tabaco, entre os adolescentes e jovens indígenas, pois é o que está mais disponível nos comércios próximos a comunidade. Assim as respostas foram agrupadas e analisadas em duas categorias: o percentual de fumantes entre os adolescentes e jovens fumantes, e os fatores relacionados à prática do tabagismo.

\section{Percentual de adolescentes e jovens fumantes na comunidade}

A Tabela 1 demonstra a faixa etária dos adolescentes e jovens que praticam o tabagismo. Ainda na Tabela 2 evidência a quantidade de cigarros ou tabaco consumido por dia.

Tabela 1 - Descrição dos entrevistados por faixa etária.

\begin{tabular}{cc}
\hline Idade & № \\
\hline $12-14$ & 25 \\
$15-17$ & 34 \\
$18-20$ & 14 \\
$21-23$ & 23 \\
$24-28$ & 17 \\
\hline Total & $\mathbf{1 1 3}$ \\
\hline
\end{tabular}

Fonte: André MFS, et al., 2020. 
De acordo com os dados da Tabela 2 é possível calcular que dos 113 adolescentes e jovens entre 12 e

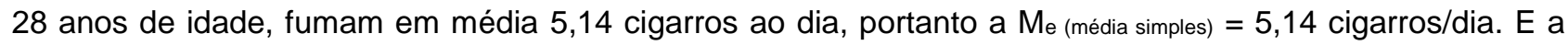
moda, representa o número mais frequente de consumo de cigarro, que seria 4 cigarros ao dia, ou seja, $M_{\circ}$ $($ moda $)=4$.

Tabela 2 - Quantidade de cigarros ou tabaco consumido por dia.

\begin{tabular}{cc}
\hline \multicolumn{2}{c}{ Quanto de cigarro ou tabaco fumam por dia? } \\
\hline Cigarro ou tabaco consumido por dia & Quantidade $\left(\mathbf{n}^{\circ}\right)$ \\
\hline 3 & 20 \\
4 & 29 \\
5 & 27 \\
6 & 25 \\
10 & 12 \\
\hline
\end{tabular}

Fonte: André MFS, et al., 2020.

O Gráfico 1 comprova a evolução do tabagismo em relação à idade do grupo de adolescente, em um grau muito alto, com $65 \%$ de consumo do tabagismo, observando que a proporção é menor na classe dos jovens, com $35 \%$.

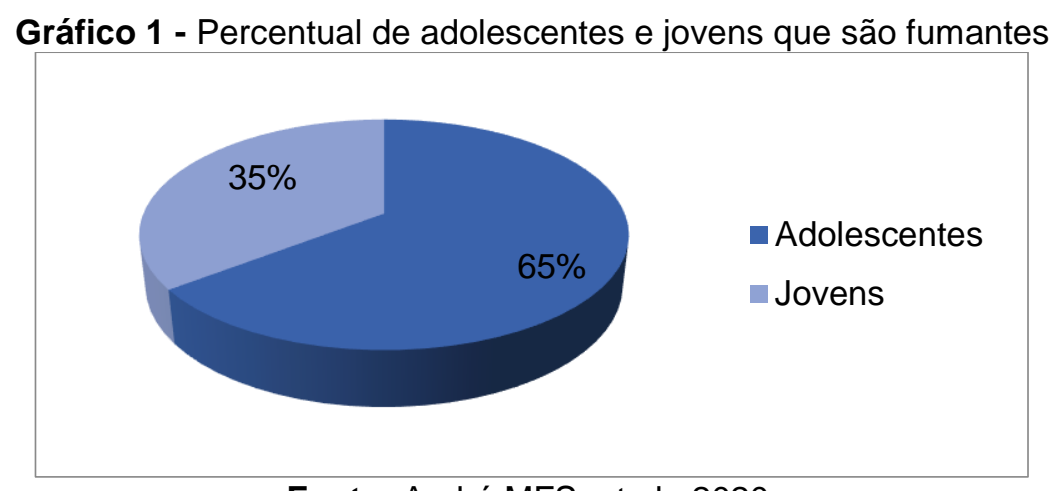

Fonte: André MFS, et al., 2020.

\section{Fatores relacionados ao tabagismo entre os adolescentes e jovens}

O Gráfico 2 exibe os principais fatores relacionados à experimentação do tabagismo entre os a adolescentes e jovens da comunidade indígena, assim citados: amigos fumantes, bebida alcoólica, convívio familiar, curiosidade, desejo, futebol, pais fumantes e a pescaria. Observa-se que os mais citados foram amigos fumantes, a bebida alcoólica, e a pescaria ficando por último dos mais citados.

Gráfico 2 - Fatores relacionados ao tabagismo entre os adolescentes e jovens.

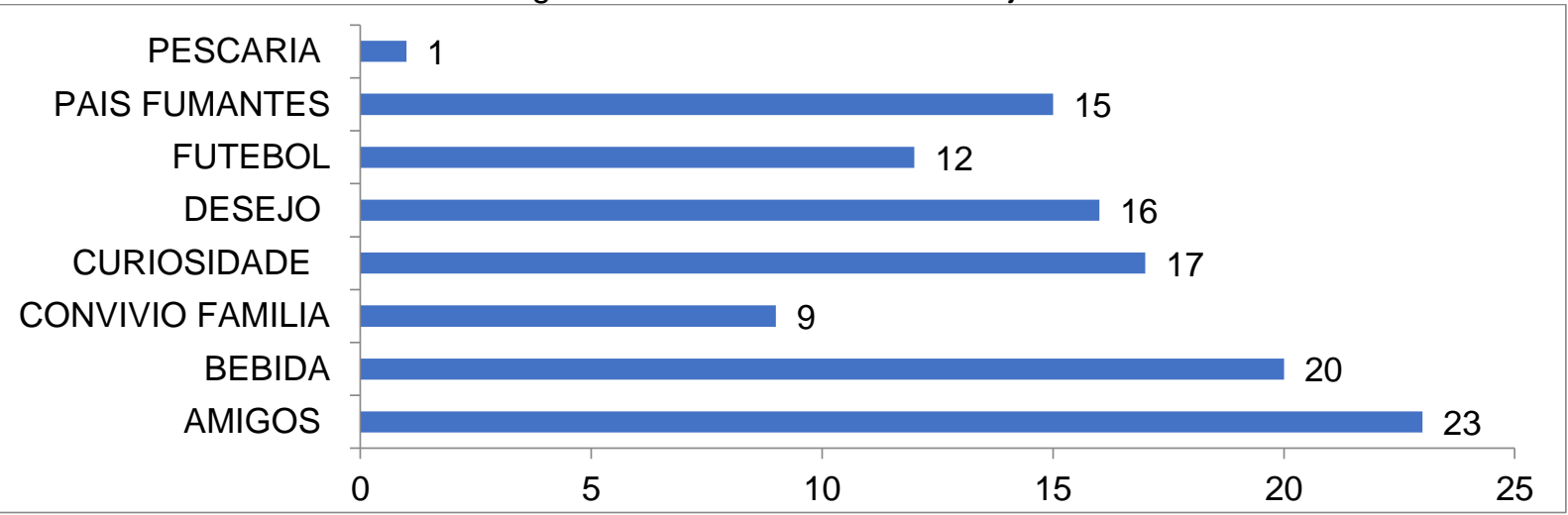

Fonte: André MFS et al., 2020. 
A maioria referiu que experimentou e continua consumindo cigarros comuns ou tabaco por influência dos amigos. E o segundo fator mais citado foi o uso do cigarro associado ao consumo de bebida alcoólica, que favorece desejo em consumir o cigarro, pois segundo os participantes o cigarro os deixam relaxados, menos estressados e eles sentem uma sensação prazerosa. E o terceiro fator seria a curiosidade, o desejo de conhecer algo novo, infringir regras e o que é proibido. O quarto seria o desejo por prazer; e o quinto seria ter pais fumantes, o que facilita o consumo do cigarro em casa. O sexto seria a diversão, como futebol. Os outros poucos relevantes estão relacionados ao convívio com suas famílias que as vezes é estressante, difícil.

\section{DISCUSSÃO}

Este estudo foi realizado a partir da percepção dos moradores da comunidade em que, percebeu-se a prática do tabagismo entre os adolescente e jovens como um agravo à saúde e era preciso buscar estratégias para entender a causa do problema e pensar em alternativas para favorecer a cessação do tabagismo entre o grupo estudado. Observou-se que os jovens começam a fumar o cigarro precocemente e vários fatores estão relacionados (por influência dos amigos, para ser aceito no grupo, como forma de socialização; o uso de bebidas alcoólicas; a curiosidade, a vontade de conhecer algo novo; o desejo por prazer, por relaxamento; sensação de poder por infringir regras; ociosidade; pais fumantes; problemas familiares, entre outros), mas são fatores que se conectam com a fase da adolescência e juventude.

A adolescência é uma fase complexa da vida, em que buscamos conhecer tudo que nos é proibido. A maioria dos jovens que se expõem, nem ao menos se importa com os danos que poderão surgir. Uma das atitudes de risco que se pode citar, é o consumo de cigarro (URRUTIA-PEREIRA, M et al., 2017). Porém, não se pode generalizar que todo adolescente que degusta um cigarro pela primeira vez, transforma-se em um fumante. No entanto, o início da prática do tabagismo está na experimentação do primeiro cigarro. Em razão das alterações de comportamento que ocorrem na adolescência e a exposição a inúmeras circunstâncias de risco, como a experiência ao tabaco, revela que está fase do ciclo da vida merece uma grande atenção (SILVA RMA, et al., 2019).

Santos VP, et al. (2021) chamam a atenção para se conhecer o território em que as necessidades e os problemas de saúde acontecem. Assim, com o propósito de estudar as implicações que o hábito de fumar pode causar à saúde das populações humanas, se faz necessário uma análise sobre o perfil de tabagismo do local amostral examinado. Hoje em dia, compreende-se que múltiplos fatores estão conexos ao consumo e cessação do hábito de fumar.

Quando o cigarro é queimado pode-se identificar diversas substâncias químicas, 43 destas são substâncias cancerígenas. A nicotina é mais enfatizada entre elas, pela razão de ser um excitante do sistema nervoso central que provoca uma sensação prazerosa, de bem-estar e descontração em seus consumidores, mas é considerada o principal elemento relacionado a evolução para dependência (BRUSTOLIN M, et al., 2019).

A percepção do cigarro como uma prática proibida excita a cobiça do adolescente e do jovem de desobedecer e suas principais razões para fumar são as aspirações, seria não ser mais criança e proclamarse adulto e de se estabelecer no grupo. Devido às formas de ser e de se comportar, os adolescentes surgem com uma maior vulnerabilidade para os planos da indústria tabagista e à publicidade (MINISTÉRIO DA SAÚDE, 2020).

O estudo de Figueiredo VC, et al. (2016) sobre a prevalência de tabagismo entre adolescente, corrobora com os resultados encontrado em nosso estudo, no que concerne ao maior percentual de adolescentes que são fumantes na comunidade que foi na faixa etária de 15 a 17 anos. Entre os jovens, a prevalência de tabagismo quase sempre aumenta com a idade, no entanto, os resultados encontrados mostraram a evolução do tabagismo a partir de idades precoces, sendo a incidência na faixa etária de 24 a 28 anos, bem menor.

As áreas rurais são afetadas pela ausência/escassez de opções de lazer e cultura, prática de esportes e ambientes de convívio públicos, o que pode implicar negativamente na vida dos adolescentes e contribuir para adoção de hábitos de vida não saudáveis, como o tabagismo (MINISTÉRIO DA SAÚDE, 2010). Vale ressaltar, que a comunidade fica a $320 \mathrm{~km}$ da cidade Boa Vista, onde as oportunidades de estudo, emprego, lazer, entre outros são muito difíceis. 
Em relação aos amigos fumantes, existem evidências de que se trata de um dos mais importantes fatores de risco para a iniciação ao tabagismo, verificando que adolescentes não fumantes que têm amigos que o fazem, possuem chances maiores de, no futuro, começar a fumar (FARIA BL, et al., 2019). Um dos fatores mais citados foi ter amigos fumantes, que podem influenciar no início do tabagismo.

Segundo alguns estudos como Sirqueira RS, et al. (2020), Werneck FA, et al. (2016) e Almeida JP, et al. (2011) afirmam que existe uma importante relação entre álcool e o cigarro o que favorece, o uso e abuso da prática do tabagismo. Neste sentido, estes achados corroboram com nossa pesquisa, em que foi evidenciada a necessidade de fumar anexa ao uso de bebidas alcoólicas entre os entrevistados.

A sensação de prazer e relaxamento ocasionada pelo cigarro pode motivar o uso contínuo do cigarro. (WILLEMANN JR e BURCI LM, 2014). A curiosidade; a percepção de poder ao provar o tabaco e as implicações pelo seu uso; a persuasão dos amigos e problemas familiares; Um padrão vendido pela mídia, publicidade, músicas, uma imaginação de popularidade; a falta de informações dos familiares quanto aos componentes químicos, e os malefícios do cigarro para seus filhos, unidos com a falta de cuidado dos pais na criação dos filhos, em que na maioria das vezes é permeada por agressividade e repressão são os principais determinantes para o início prematuro do tabagismo (TEIXEIRA CC, et al., 2017; WILLEMANN JR e BURCI LM, 2014).

A prática do tabagismo começa na fase da adolescência, em que somos motivados a descobrir coisas novas, contudo na busca de pertencimento, amigos e ser aceito em um grupo, entende-se que o uso do cigarro significa um modo de vida contemporâneo, pela regularidade e anuência da sociedade ao uso do cigarro (GIRON MPN, et al., 2010). Improvisando uma análise sobre os fatores, eles aceitam que é para se sentirem aceitos dentro do grupo, fazendo qualquer atividade.

A prática do tabagismo entre os adolescentes e jovens indígenas é uma realidade, e vem aumentando, os deixando vulneráveis a vários problemas de saúde. Como é confirmado no estudo de Nascimento VN, et al. (2021), hábito de fumar entre os mais jovens cresce cada vez mais, os deixando expostos ao vicio, atingindo sua saúde. Os autores observaram que o uso do tabaco nos jovens começa de forma esporádica "como um Hobby ou para diminuir o estresse" e evoluem para a dependência química por tempo indeterminado. Pela gravidade do problema, principalmente entre os povos indígenas, se faz necessário que as autoridades sanitárias do nosso país busquem táticas para amenizar a situação, como por exemplo melhorar a fiscalização sobre a mídia e a comercialização do produto ou criar leis que possam impedir a sua comercialização para os mais vulneráveis.

Atualmente, o tabagismo é considerado um estilo de vida para os adolescentes e jovens sendo umas das principais causas de doenças. $O$ uso do cigarro pode desenvolver principalmente câncer, doenças cardiovasculares, pulmonares, entre outras (NUNES SOB, et al., 2011). Os fumantes passivos e ativos, com o passar do tempo, podem sofrer vários tipos de malefícios, como por exemplo, neoplasias no sistema respiratório, digestivo e urinário, e estão mais vulneráveis a doenças cardíacas e acidentes vasculares cerebrais (RIBEIRO FAC, et al., 2015). Na comunidade, observa-se que a maioria dos participantes sabem e conhecem sobre os malefícios do tabagismo, os mais citados foram: o aborto, câncer não identificado, cansaço, câncer no pulmão, dificuldade respiratória, enfraquecimento do organismo, fraqueza, tontura e vício.

\section{CONCLUSÃO}

Conclui-se que existe uma prática elevada de tabagismo entre os adolescentes e jovens da comunidade indígena. Os participantes entendem que o tabagismo é uma doença, e a possibilidade de disfuncionalidades futuras. Ressalta-se que a associação de bebida alcoólica e o tabaco, pode ocasionar problemas sérios de saúde, sociais/financeiros, e dentre as diversas razões que favorecem o início precoce do tabagismo entre os adolescentes e jovens indígenas, destacamos a influência dos amigos, o uso de bebidas alcoólicas, a procura por prazer, diversão e por ter pais tabagistas. Vale lembrar que se faz necessário mais estudos sobre 0 tabagismo entre jovens indígenas da região norte do país e do estado de Roraima, para que se possa prevenir os malefícios decorrentes dessa prática e para produzir conhecimentos sobre essa temática. 


\section{AGRADECIMENTOS E FINANCIAMENTO}

Agradecemos à Universidade Federal de Roraima pelo financiamento para publicação deste artigo. Ao Instituto Insikiran de Formação Superior Indígena, todos os seus professores, estudantes e servidores e aos povos indígenas de Roraima pelo apoio e suas contribuições.

\section{REFERÊNCIAS}

1. ALMEIDA JP, et al. Prevalência e características do tabagismo na população universitária da região de Lins-SP. Revista Brasileira de Enfermagem, 2011; 64(3): 545-50.

2. ALVES EC, et al. Análise comparativa do pico de fluxo expiratório de universitários saudáveis, obesos e tabagistas. Revista Eletrônica Acervo Saúde, 2014; Sup.1: 63-69.

3. ASSIS SG, et al. O tema da adolescência na saúde coletiva - revisitando 25 anos de publicações. Ciência \& Saúde Coletiva [online], 2020; 25(12): 4831-4842.

4. BARRETO SM, et al. Experimentation and use of cigarette and other tobacco products among adolescents in the Brazilian state capitals (PeNSE 2012). Revista Brasileira de Epidemiologia [online], 2014, v. 17, suppl 1, 62-76.

5. BORRACI RA, MULASSI AH. Tobacco use during adolescence may predict smoking during adulthood: simulation-based research. Archivos Argentinos de Pediatría. 2015; 113(2): 106-113.

6. BRUSTOLIN M, et al. Eficácia do tratamento do tabaqismo na perspectiva da redução de danos e do cuidado farmacêutico. Revista Eletrônica Acervo Saúde, 2019; 11(17): e1565.

7. BUENO SR. O TABAGISMO NA ADOLESCÊNCIA: uma proposta de intervenção educativa. Ibati, 2011.

8. BRASIL. Lei no 8.069 , de 13 de julho de 1990. Dispõe sobre o Estatuto da Criança e do Adolescente dá outras providências. Disponível em: https://www.gov.br/mdh/pt-br/centrais-de-conteudo/crianca-e-adolescente/estatuto-da-crianca-e-doadolescente-versao-2019.pdf. Acesso em: 24 de jan. de 2022.

9. BRASIL. Lei $n^{\circ} 12.852$ de 5 de agosto de 2013. Institui o Estatuto da Juventude e dispõe sobre os direitos dos jovens, os princípios e diretrizes das políticas públicas de juventude e Sistema Nacional de Juventude (SINAJUVE). Disponível em: https://www.prattein.com.br/home/images/stories/Juventude/Estatuto_da_Juventude.pdf. Acesso em: 24 de jan. de 2022.

10. CARIOCA AT, et al. Percepção sobre a Diabetes Mellitus 2 na comunidade indígena Jabuti Bonfim - RR: um relato de experiência. Revista Eletrônica Acervo Saúde, 2021; 13(2): e6000.

11. FARIA BL, et al. Prevalência e fatores associados à experimentação do cigarro entre escolares de uma cidade do interior de Minas Gerais. Revista Científica da Faculdade de Medicina de Campos. 2019; 14(1).

12. FIGUEIREDO VC, et al. ERICA: prevalência de tabagismo em adolescentes brasileiros. Revista de Saúde Pública. 2016; 50(supl 1): 12s.

13. FILHOC BB. et al. Tabagismo no Brasil: impacto econômico na saúde pública e seu tratamento. Revista Eletrônica Acervo Médico, 2021; 1(1): e9043.

14. GIL AC. Como elaborar projetos de pesquisa. [2. Reimpr.]. - 6. ed. São Paulo: Editora Atlas, 2018; 92-102.

15. GIRON MPN, et al. Prevenção do tabagismo na adolescência: um desafio para a enfermagem. Revista Mineira de Enfermagem, 2010; 14(4): 587-594.

16. INSTITUTO NACIONAL DE CÂNCER (INCA). 2020. Causas e prevenção. Crianças, adolescentes e jovens. Disponível em: https://www.inca.gov.br/tabagismo/criancas-adolescentes-jovens. Acessado em: 9 de nov. de 2020.

17. KONKIEWITZ EC (Organizadora). Aprendizagem, comportamento e emoções na infância e adolescência: uma visão transdisciplinar. Dourados-MS: Ed. UFGD, 2013. 312 p. Disponível em: https://repositorio.ufgd.edu.br/jspui/handle/prefix/1047. Acessado em: 24 de jan. de 2022.

18. MINISTÉRIO DA SAÚDE. Secretaria de Atenção em Saúde. Departamento de Ações Programáticas Estratégicas. Diretrizes nacionais para a atenção integral à saúde de adolescentes e jovens na promoção, proteção e recuperação da saúde. Brasília https://bvsms.saude.gov.br/bvs/publicacoes/diretrizes_nacionais_atencao_saude_adolescentes_jovens_promocao_saude.p df. Acessado em: 24 de ian. de 2021.

19. NASCIMENTO VN, et al. Tabagismo na juventude: uma revisão bibliográfica. Revista Artigos. Com, 2021; 26 : e6693.

20. NUNES SOB, et al. Tabagismo, comorbidades e danos à saúde. In: NUNES SOB e CASTRO MR (Org.). Tabagismo: Abordagem, prevenção e tratamento [online]. Londrina: Editora da Universidade Estadual de Londrina (EDUEL), 2011. pp. 17-38.

21. RIBEIRO FAC, et al. Percepção dos pais a respeito do tabagismo passivo na saúde de seus filhos: um estudo etnográfico. Revista Paulista de Pediatria. 2015; (4): 394-399.

22. SANTOS VP, et al. Perfil de tabagismo e frequência de micronúcleos em indivíduos de um município do Sul de Minas Gerais. Revista Eletrônica Acervo Saúde, 2021; 13(2): e5621.

23. SILVA RMA, et al. Experimentação de tabaco e fatores associados entre adolescentes da zona rural de Vitória da Conquista, BA, Brasil. Ciência \& Saúde Coletiva, 2019; 24(2): 431-441.

24. SILVA ST, et al. Combate ao Tabaqismo no Brasil: a importância estratégica das ações governamentais. Ciência \& Saúde Coletiva [onlinel. 2014; 19(2): 539-552.

25. SIRQUEIRA RS, et al. Perfil do uso do tabaco em estudantes de medicina em uma universidade particular de Sergipe. Revista Eletrônica Acervo Saúde, 2020; 48: e3371.

26. TEIXEIRA CC, et al. Fatores relacionados à iniciação tabágica em adolescentes. Revista Mineira de Enfermagem. 2017; 21 : e1039.

27. URRUTIA-PEREIRA $M$, et al. Prevalência e fatores associados ao tabagismo entre adolescentes. J. Jornal de Pediatria [online]. 2017, 93(3): 230-237.

28. WERNECK FA, et al. Prevalência do tabagismo entre os estudantes de Medicina da Universidade Severino Sombra. Revista de Saúde. 2016; 7(2): 08-11.

29. WORLD HEALTH ORGANIZATION (WHO). 2021. Tobacco. Disponível em: https://www.who.int/news-room/factsheets/detail/tobacco. Acessado em: 26 de dez. de 2021.

30. WORLD HEALTH ORGANIZATION (WHO). 1986. Young People's Health - a Challenge for Society. Report of a WHO Study Group on Young People and Health for All. Disponível em: https://apps.who.int/iris/handle/10665/41720. Acessado em: 24 de jan. de 2021.

31. WILLEMANN JR, BURCI LM. Os malefícios do uso do cigarro e seu impacto na sociedade. Revista Gestão \& Saúde. 2014; 11: 28-34. 